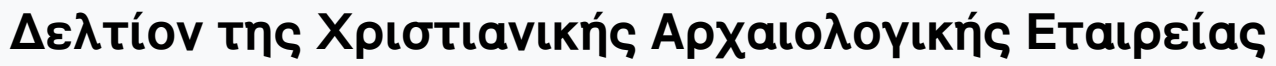

Tóp. 4 (1939)

$\Delta \varepsilon \lambda$ tíov XAE 4 (1936-1938), Пврі́обос Г'

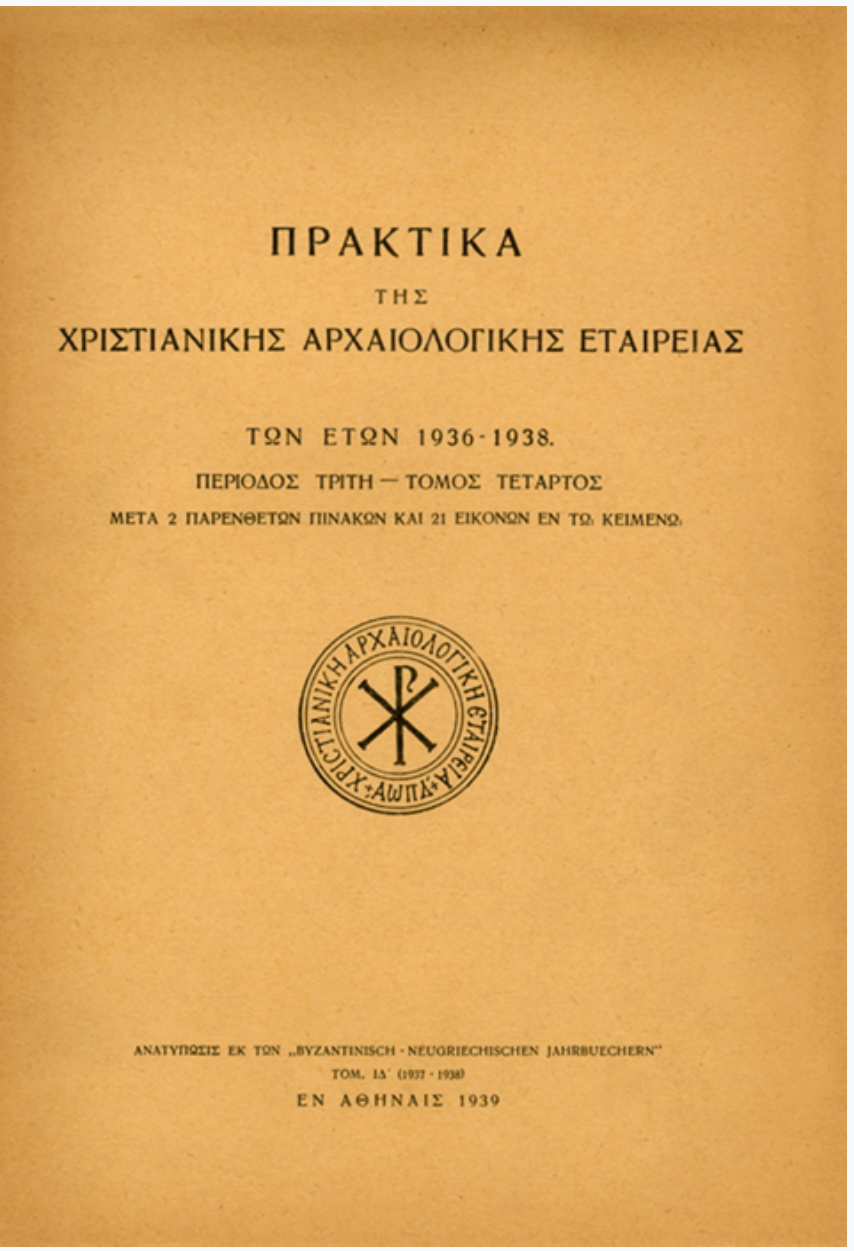

Textkritisches zur Vita des Nikolaus Studites

Theodor NISSEN

doi: $\underline{10.12681 / \text { dchae. } 1425}$

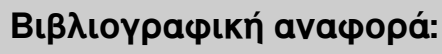

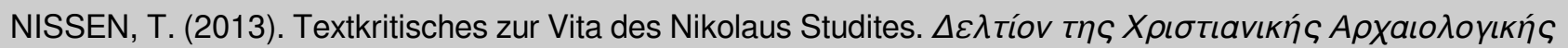
Eтaısía ৎ, 4, 91-99. https://doi.org/10.12681/dchae.1425 


\section{$\triangle \mathrm{E} \Lambda \mathrm{TION}$ TH $\Sigma$ XPI $\Sigma$ TIANIKH $\Sigma$ APXAIO $\Lambda$ OГIKH $\Sigma$ ETAIPEIA $\Sigma$}

Textkritisches zur Vita des Nikolaus Studites

Theodor NISSEN

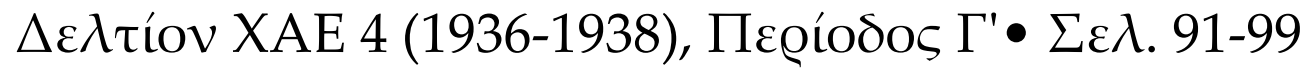

A@HNA 1939 


\title{
TEXTKRITISCHES ZUR VITA DES NIKOLAUS STUDITES
}

\author{
VON \\ THEODOR NISSEN \\ (KIEL) *
}

Eine anonyme Vita des Nikolaus Studites ( $\dagger$ 4. Februar 868) hat Fr. Combefis, Patrum bibl. novum auctarium II (Paris 1648) col. 889-953 aus einem codex regius ${ }^{1}$ herausgegeben (zitiert wird nach dem Abdruck bei Migne, Patr. Gr. 105, col. 864-925). Sie ist von einem Mönch des Studiten-Klosters 40 Jahre nach des Abtes Nikolaus zweitem Nachfolger. Hilarion verfaßt, als Kreta noch von den Arabern beherrscht wurde, also vor deren Vertreibung durch Nikephoros Phokas 961 (s. Ernst Kirsten, Die Antike 14, 1938, S. 334). Was die Vita für die Kirchengeschichte bedeutet, hat Ernst v. Dobschütz in der Byz. Zeitschrift 18 (1909) S. 70-72 dargelegt. Ihr Verfasser heißt bei Combefis nicht mit Unrecht eruditus nec inelegans (im ,Monitum“ 863/4); ich verweise dafür auf die von Combefis

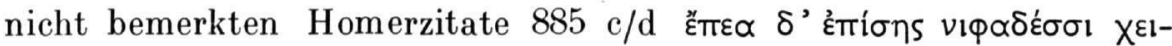

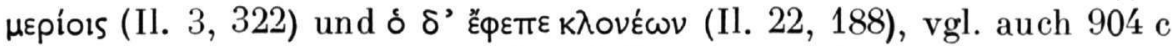

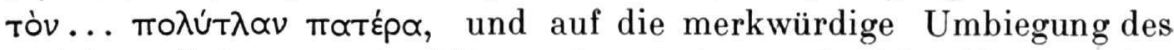

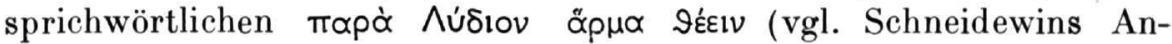
merkung zu Gregor. Cypr. II, 99, Corpus paroem. gr. I, 367 f.), die

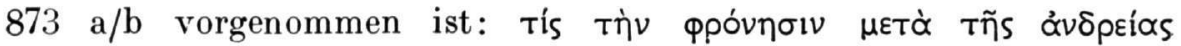

* Denkschrift der Christlich-Archäologischen Gesellschaft zu Athen durch Prof. Dr. N. A. Bees vorgelegt.

1) Sowohl der Parisinus gr. 1450 wie 1452, beide olim regii, enthalten die Vita des Nikolaus, s. Catalogus cod. hagiogr. graec. bibl. nat. Paris. edd. hagiographi Bollandiani et Henricus Omont (Bruxellis Parisiis 1896) p. 115 u. 118. Uber den Paris. gr. 1452 vgl. A. Ehrhard, Überlieferung und Bestand der hagiogr. u. homilet. Literatur der griech. Kirche I, 1 (Lpz. 1937) S. 577-580. Ehrhard hält es wegen der "Gegenwart des Festes des Abtes Nikolaus von Studion" in der Handschrift für sehr wahrscheinlich, daß sie in diesem Kloster geschrieben wurde (S. 580). 


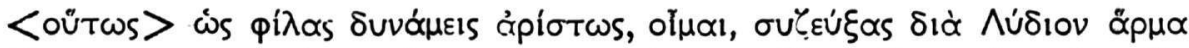

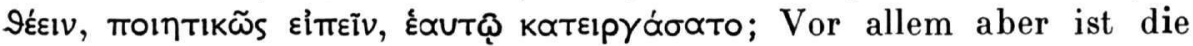
große Sorgfalt hervorzuheben, mit der der Verfasser die rhythmische Kadenz der Satzglieder gestaltet hat. Hierüber sagt Wilhelm Meyer (Der accentuierte Satzschluß usw., Göttingen 1891, S. 20= Ges. Abh. zur mittellat. Rythmik II, Berlin 1905, S. 220 f.), er habe außer der Schrift des Johannes Eleemosynarios (das Leben Tychons) und den vielen des Sophronios bisher nur in diesem Stücke ausschließlich den doppeldaktylischen Schluß mit 2 Senkungen sowohl vor als nach der letzten Hebung angewendet gefunden. Ursprünglich hatte Meyer auch den Anfang der Vita als Beispiel gegeben (S. 27 f., im Wiederabdruck $i_{n}$ den Ges. Abh. fortgefallen) und hinzugefügt, daß vor der letzten Hebung auch 4 unbetonte Silben gestattet sind. In der Tat sind bei Satzschlüssen O, 1 oder 3 Senkungen v o $r$ der letzten Hebung durchaus verpönt; bei Gliedschlüssen finden sich solche Fälle ganz vereinzelt, z. B. vor einer Begründung oder vor einem Relativsatz, weil der Verf. dann das Glied nicht als abgeschlossen betrachtet, sondern ohne Unterbrechung in das folgende übergeführt hat, vgl. $881 \mathrm{~b}$ ơ $\lambda \lambda \dot{\alpha}$

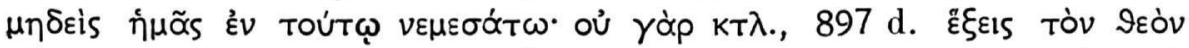

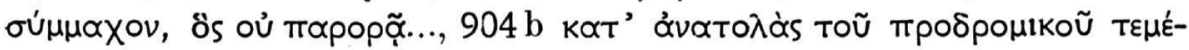
vous, ötrov $\delta \grave{~} \kappa \tau \lambda$. Satz- und Gliedschlüsse mit $O$ oder 1 Senkung nach der letzten Hebung finden sich im Ganzen etwa 15, z. B. 877 b

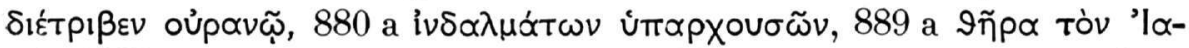

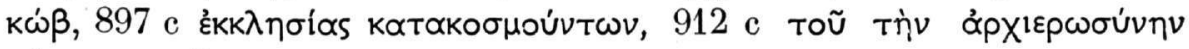

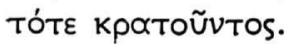

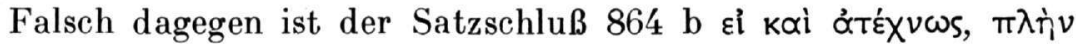

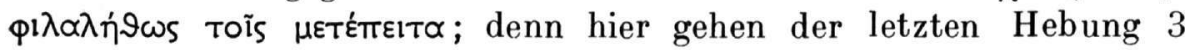

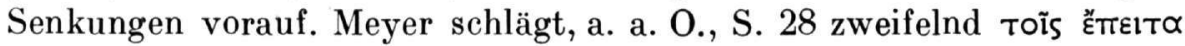
vor; mir ist es wahrscheinlicher, da $\beta$ hinter $\phi \iota \lambda \alpha \lambda \eta \dot{\eta} \omega_{s}$ ein $\pi \omega s$ ein-

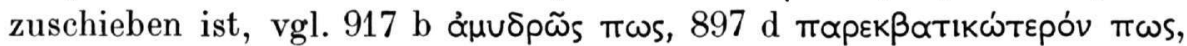

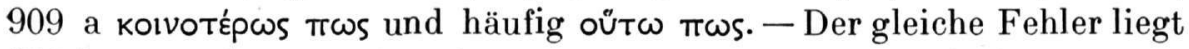

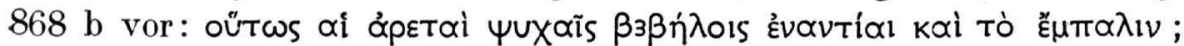

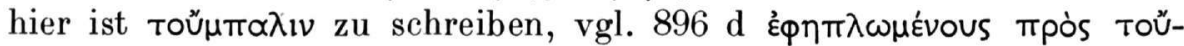

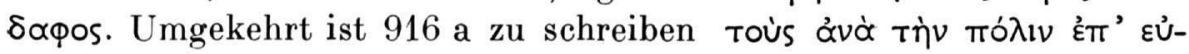

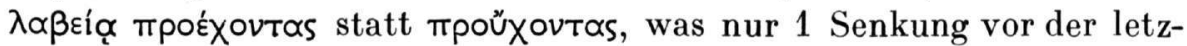
ten Hebung ergeben würde. Dieser Fehler liegt 869a vor: (Nikolaus)

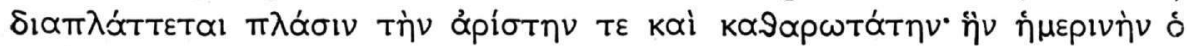

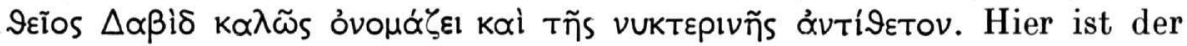
ganze Relativsacz als ein Zusatz zu streichen, der nicht anders als töricht genannt werden kann. Denn selbst wenn man aus Ps. 139, 15 und 16-eine andere Stelle kommt nicht in Frage-einen Gegensatz

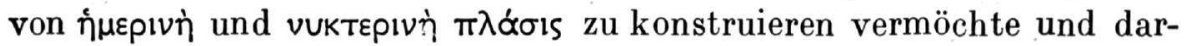




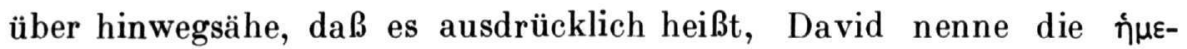

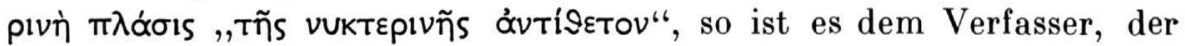
alle andern Schriftzitate sachgemäß und verständig angebracht hat, unmöglich zuzutrauen, daß er dieser Schriftstelle eine Deutung gegeben hat, die mit dem Ausdruck, grobes Mißverständnis" noch viel

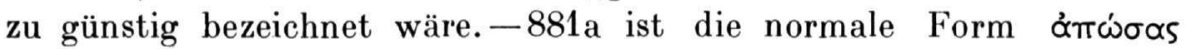

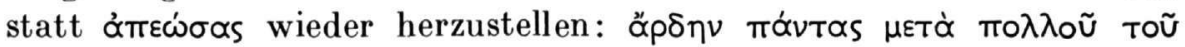

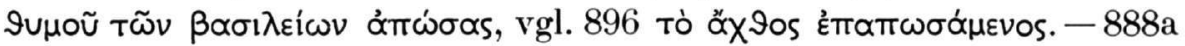

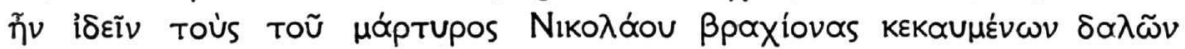

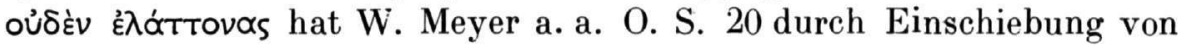

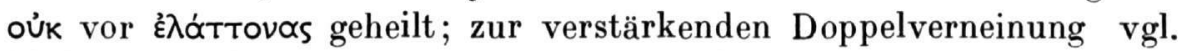

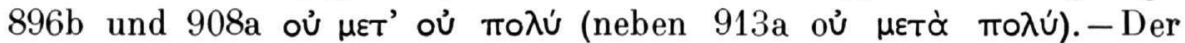

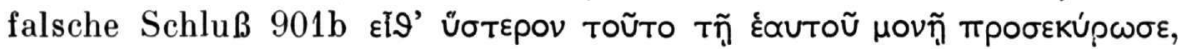

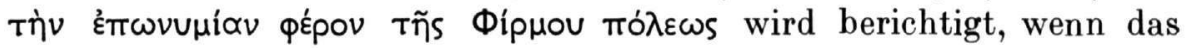

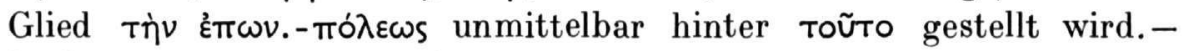

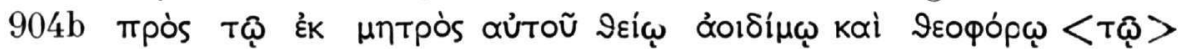

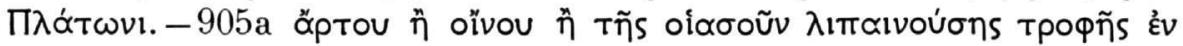

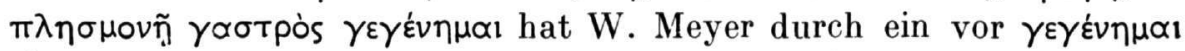
eingeschobenes ou berichtigt und damit, wohl ohne sein Wissen, die Überlieferung wiederhergestellt; denn das oủ findet sich im Text bei Combefis col. 932 und ist nur im Abdruck bei Migne irrtümlich fortgefallen ${ }^{1}$. Das Fragezeichen, das Meyer hinter oủ gesetzt hat, ist unberechtigt; alle Genitive hängen von $\pi \lambda \eta \sigma \mu \circ v \tilde{n}$ ab. $-913 \mathrm{c}$ ist $\tau \tilde{\omega} \nu \beta \alpha-$

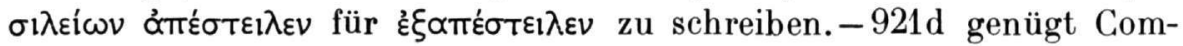

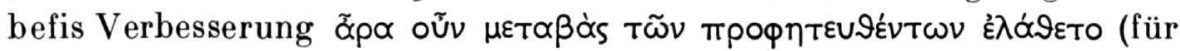
غ̇ $\lambda \alpha \dot{\tau} \tau T \omega)$ nicht nur dem Sinn, sondern bringt auch einen doppeldakty-

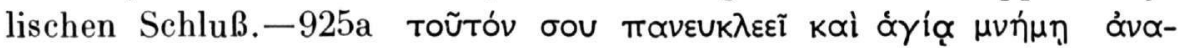

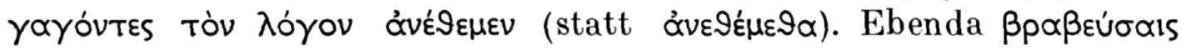

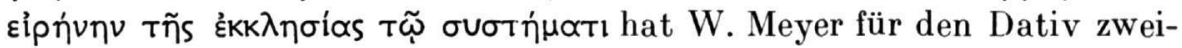
felnd тò $\sigma \dot{\sigma} \sigma \tau \eta \mu \alpha$ vorgeschlagen; sein Fragezeichen gilt vermutlich

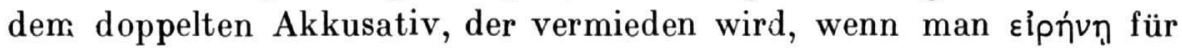

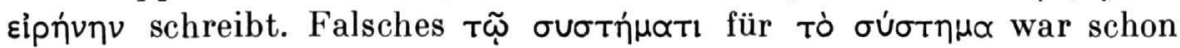
921b (

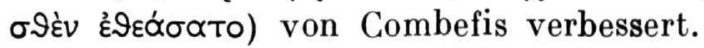

Dieser und ähnliche Fälle weisen auf die überaus große Willkür des Verfassers (oder des Schreibers?) im Gebrauch des Dativs und des Akkusativs ${ }^{2}$. Die Frage, ob und wie man hier zu verbessern

1) Nicht wenige Versehen des Combefis sind bei Migne stillschweigend berichtigt; aber es sind nicht nur viele stehngeblieben, sondern sogar neue hinzugekommen. Hier werden nur solche Fälle angeführt, die zu Zweifel und Mißverständnissen Anlaß geben könnten.

2) Vgl. hierzu Krumbacher, Ztschr. f. vgl. Sprachforschung 29 (1888) S.191 f. 
habe, wird nur in einigen Fällen durch den Rhythmus, bisweilen auch

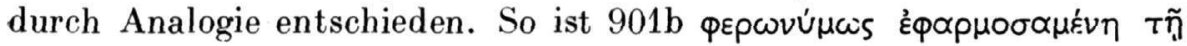

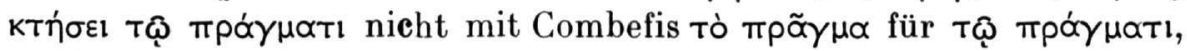

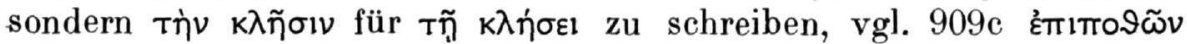

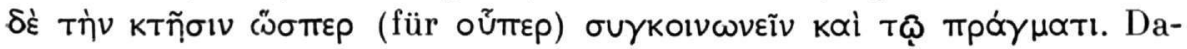

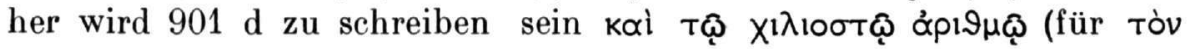

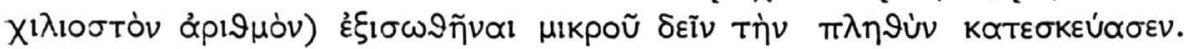

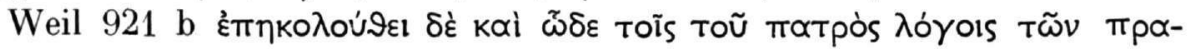

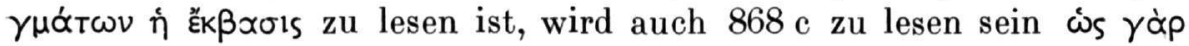

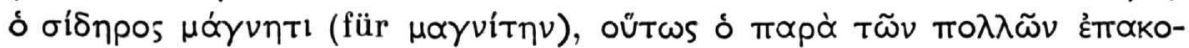

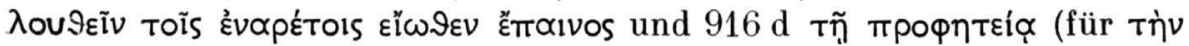

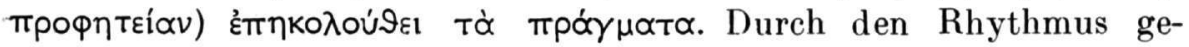

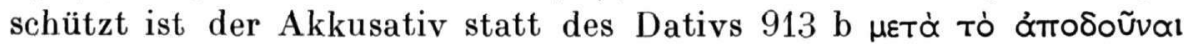
Tòv... Ipóvov tòv Ieoфópov 'I $\gamma$ vớtiov, wo also nicht mit Combefis

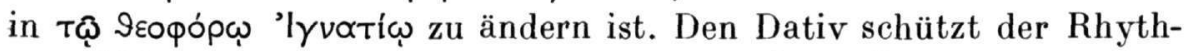

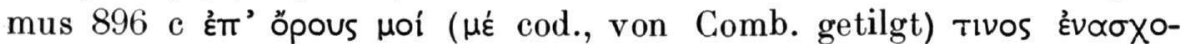

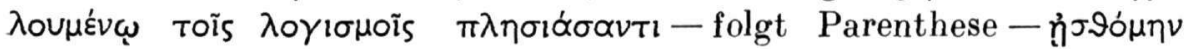

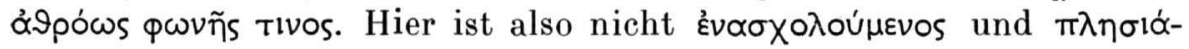
o๙s zu schreiben, wie Combefis es tut, sondern Anakoluth anzuerkennen, das der Verfasser auch sonst nicht scheut, zumal wenn da-

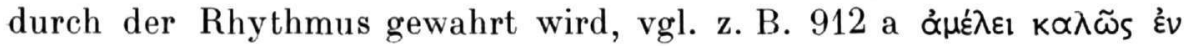

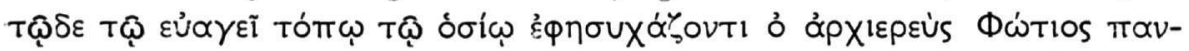

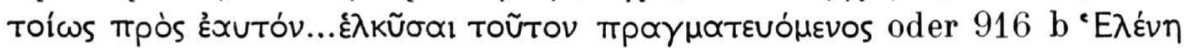

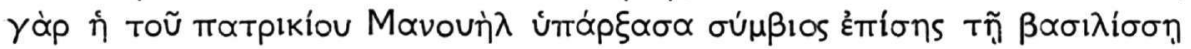

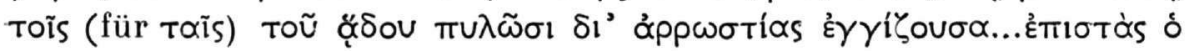

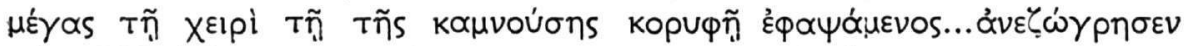

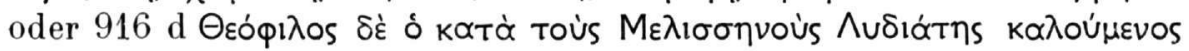

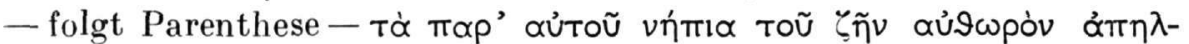
$\lambda$ á́ттоvтo. In den meisten Fällen wird man Bedenken tragen, auffällige Dative und Akkusative zu ändern, wie es Combefis getan. $\mathrm{Zu}$ beachten bleibt dabei, daß toútors und toútous in Minuskelhandschriften oft kaum zu unterscheiden ist, also braucht 864a Toútols દ̇k

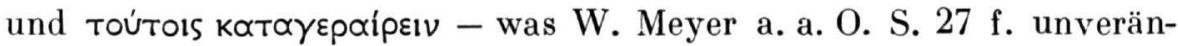
dert gelassen hat - dem Verfasser ebensowenig zugetraut zu werden

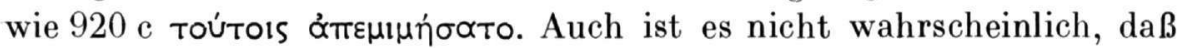

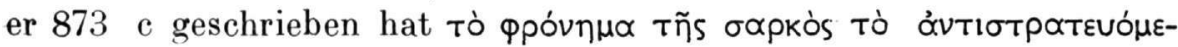

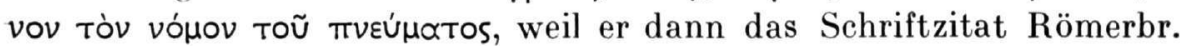
7, 23 (das bei Combefis, nicht aber bei Migne zu der Stelle vermerkt

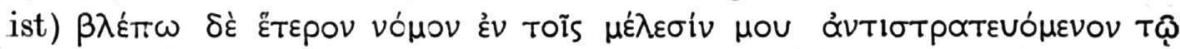

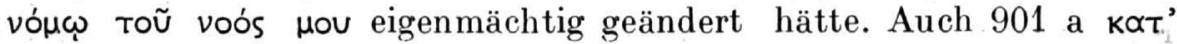

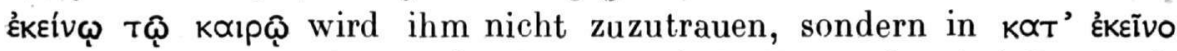

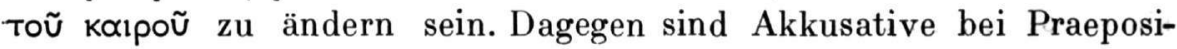




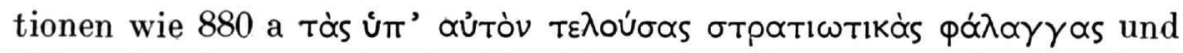

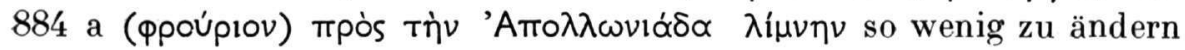

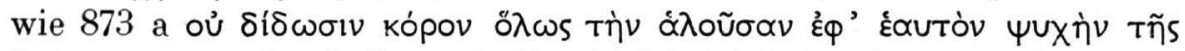

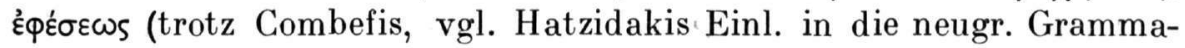

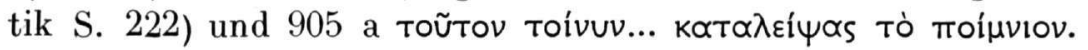

Kürzlich hat A. Debrunner festgestellt, daß den Dichtern der homerischen Gesänge die Korrektheit des Verses wichtiger gewesen sei als die der Sprache (Metrische Kürzungen und Erweiterungen bei Homer, Revue des Êtudes Indo-européennes I, 1938, S. 4 des Sonderabdrucks). Wenn man statt „des Verses" „des Rhythmus" setzt, gilt das Gleiche von den spätgriechischen Virtuosen der Kadenz (vgl. Usener, Der heilige Tychon S. 70).

Nunmehr mögen Stellen besprochen werden, bei denen eine Rücksichtnahme auf den Rhythmus nicht oder doch nicht in erster

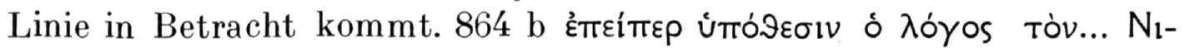

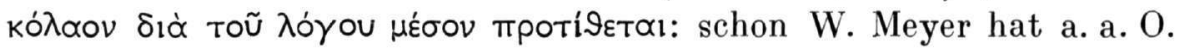

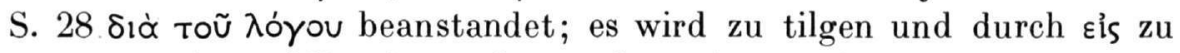

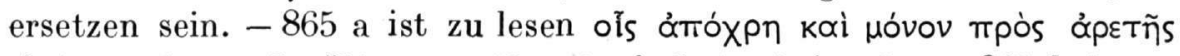

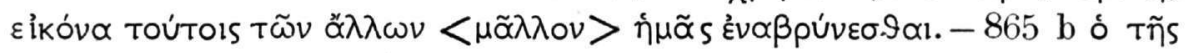

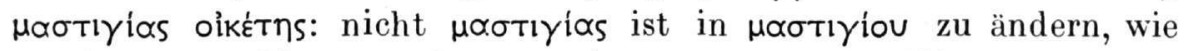

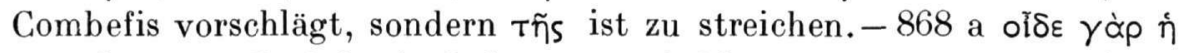

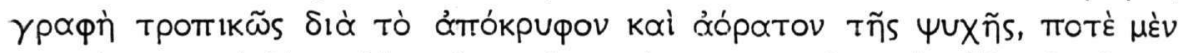

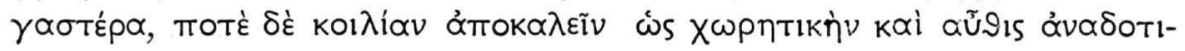

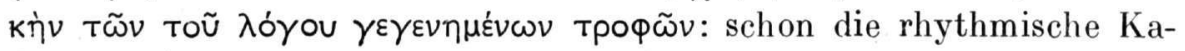

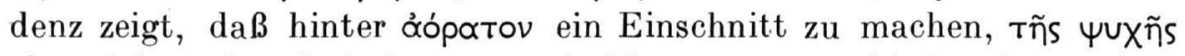

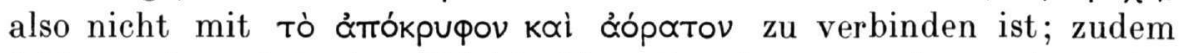

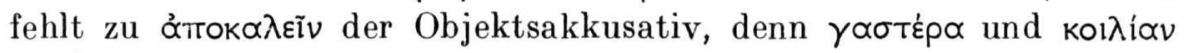

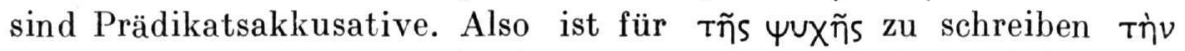

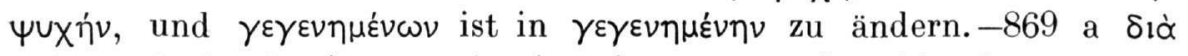

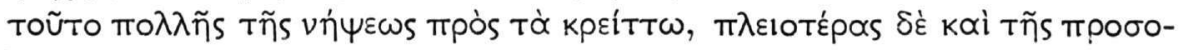

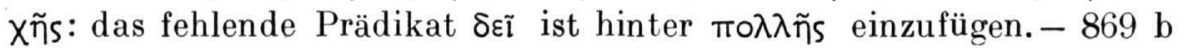

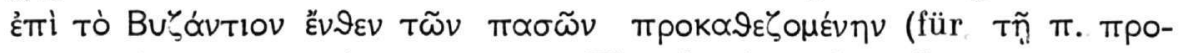

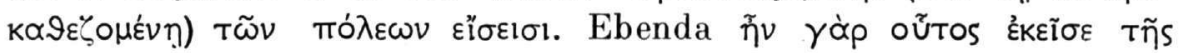

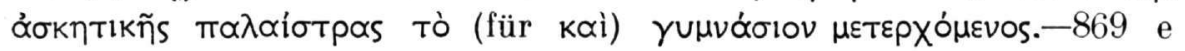
трòs tò

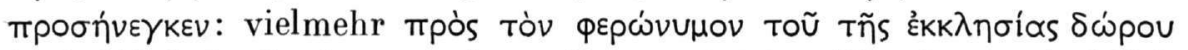

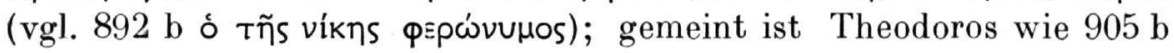

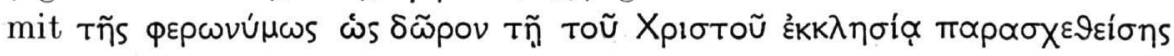

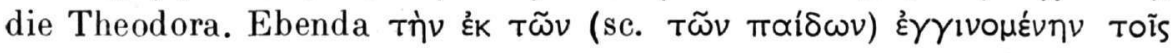

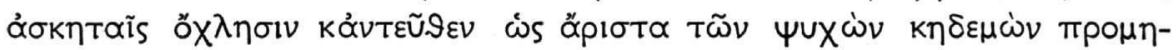

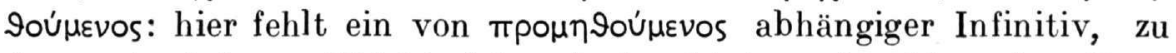

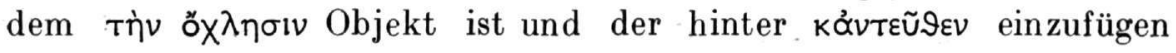




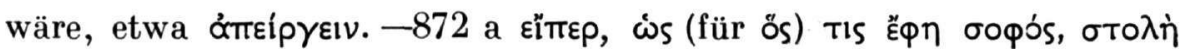
кai $\beta \tilde{\eta} \mu \alpha \kappa \tau \pi$. (Das bei Combefis falsch angegebene Zitat stammt aus Sirac. 19, 30), vgl. 873 a

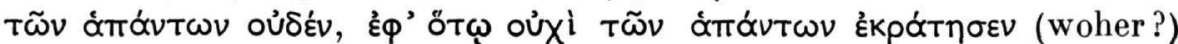

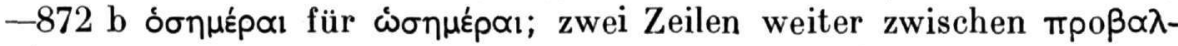

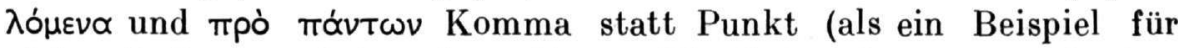
viele; die Interpunktion der edierten Vita läßt sehr zu wünschen).-

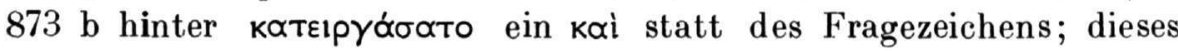

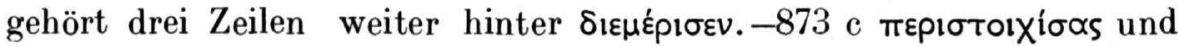

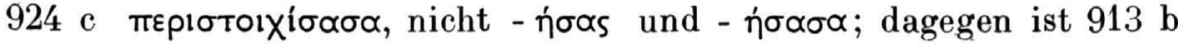

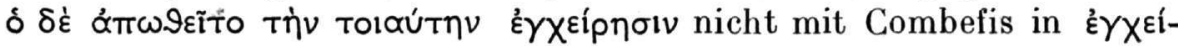

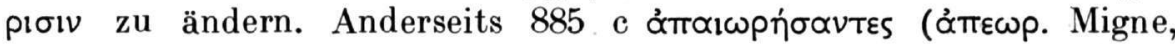

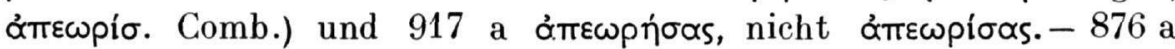

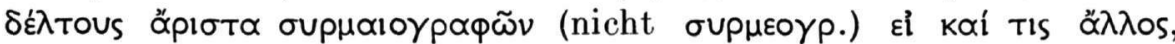

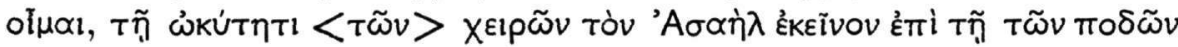

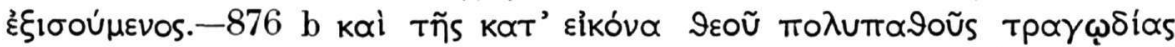

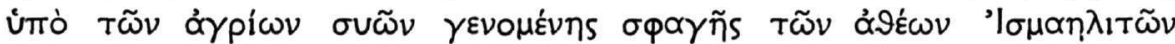

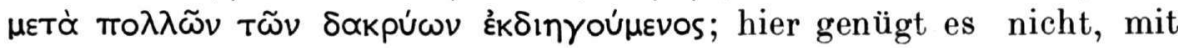

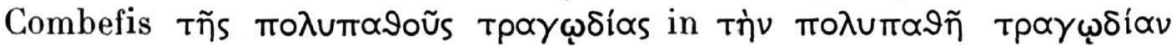

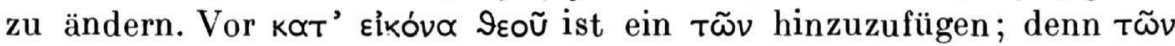

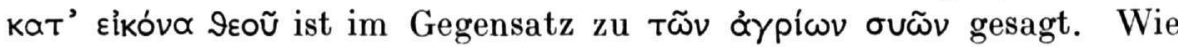

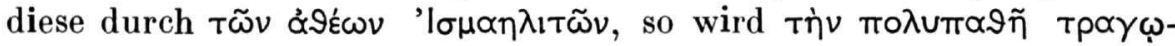

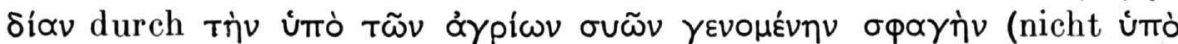

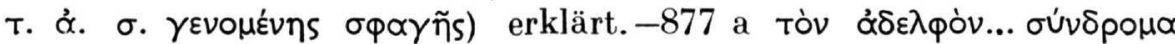

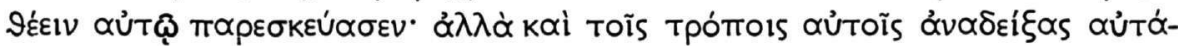

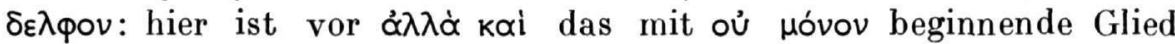

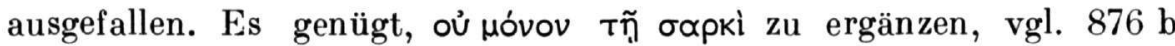

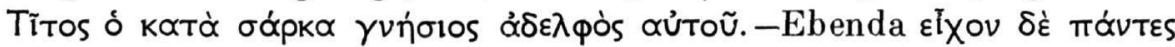

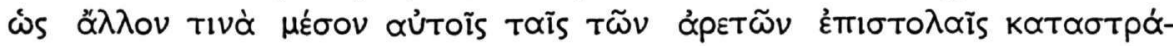

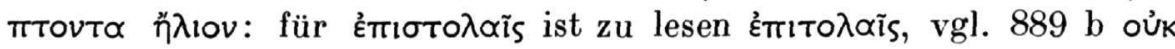

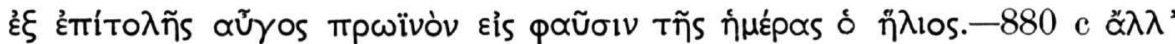

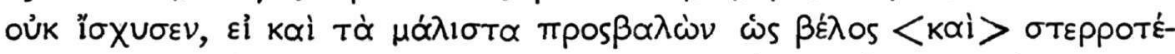

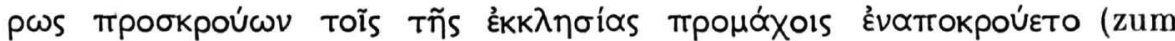
fehlenden syllabischen Augment vgl. St. B. Psaltes, Grammatik der

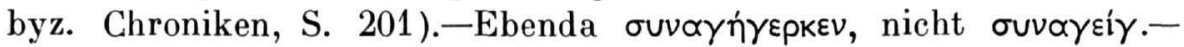

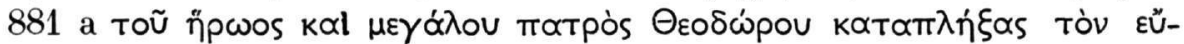

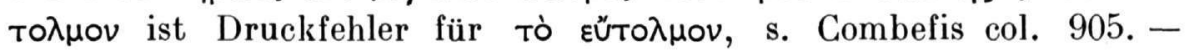

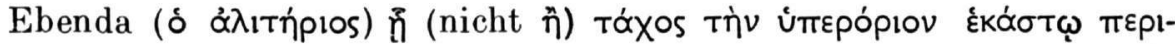

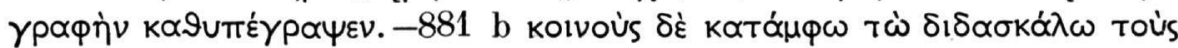

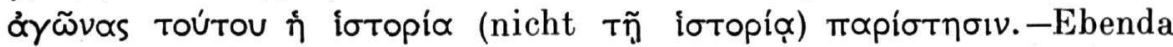

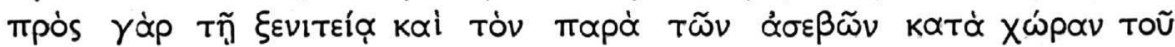

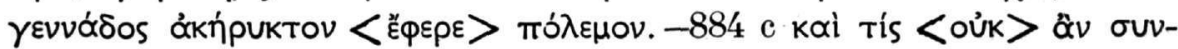




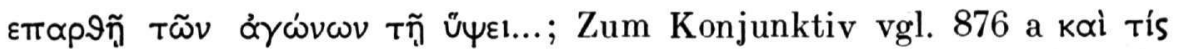

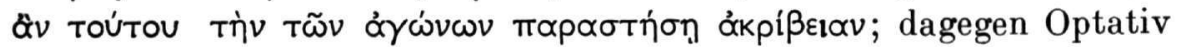

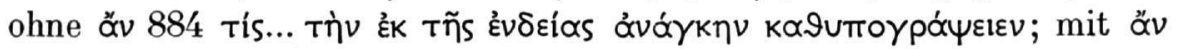

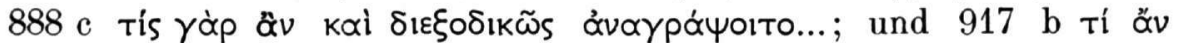

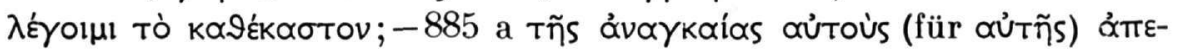

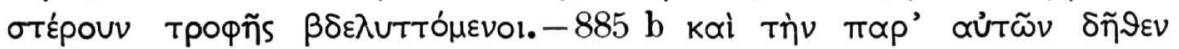

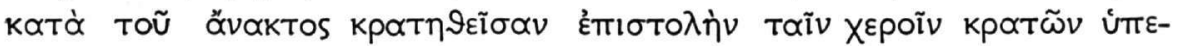

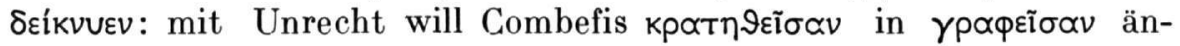

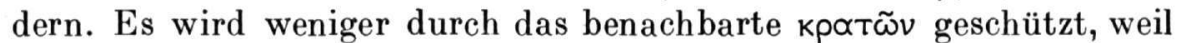
es unbeweisbar ist, daß hier kein Zufall, sondern ein beabsichtigtes

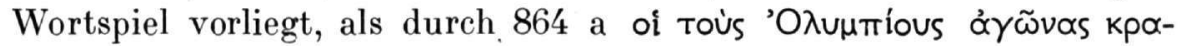

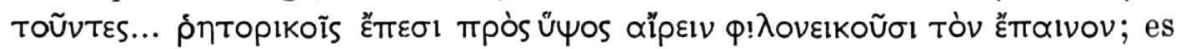
liegt also eine ähnliche Metapher vor wie beim deutschen .,in Worte fassen", ,verfassen". Weshalb Combefis weiter behauptet, daß in

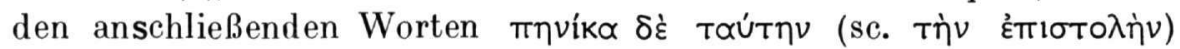

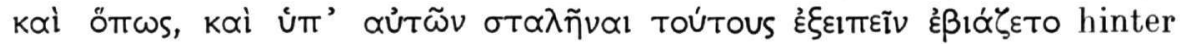

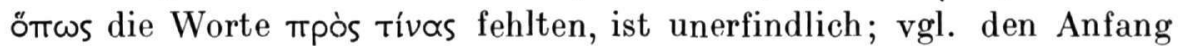

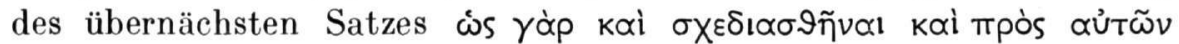

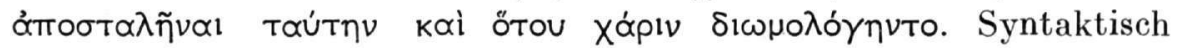
bietet die Verbindung von Acc. c. inf. und indirektem Fragesatz, dessen Verbum aus dem Infinitiv zu ergänzen ist, keine Schwierigkeit. 885 b ơ้

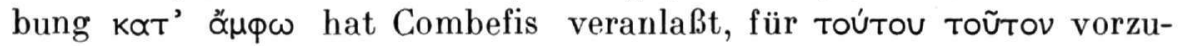

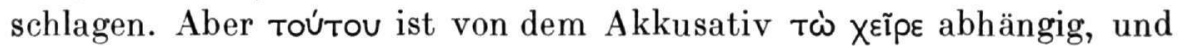

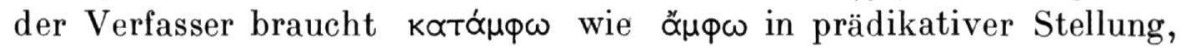

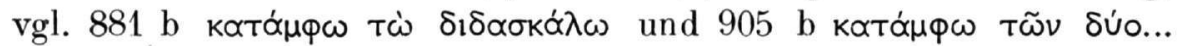

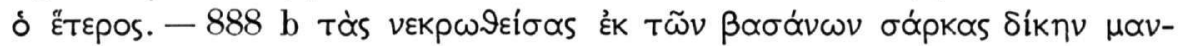

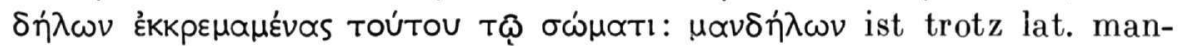
tele nicht in $\mu \alpha v \tau \eta \dot{\lambda} \omega \nu$ zu ändern, vgl. B. Meinersmann, Die lat.

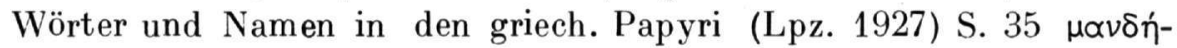

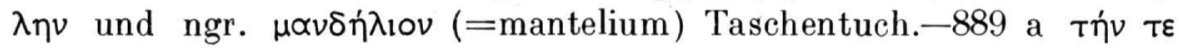

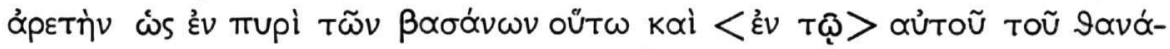

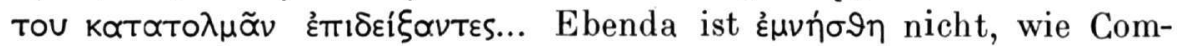
befis will, in $\mu \nu \eta \sigma Я \varepsilon i s$ zu ändern; Subjekt ist $\alpha u ̛$ 'òs d. h. Gott.-Ebenda

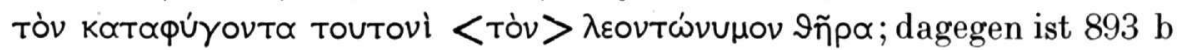

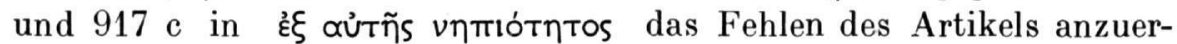

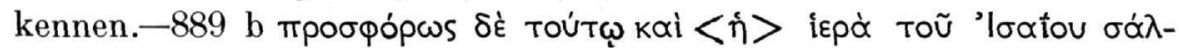

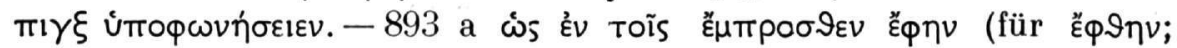
Combefis übersetzt dixi, ändert aber nicht). $-893 \mathrm{~b} \pi \alpha \rho \alpha \beta \alpha \dot{ } \lambda \lambda \varepsilon ı v$

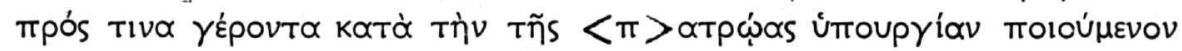

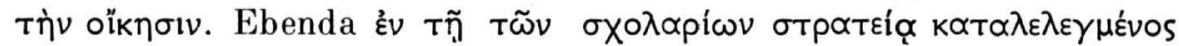

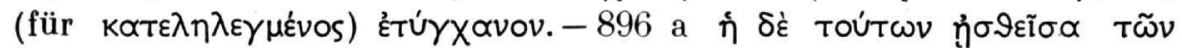


$\lambda o ́ \gamma \omega \nu$ : wenn $\eta^{\circ} \sigma \varepsilon \varepsilon i \sigma \alpha$ als $\eta_{\sigma} \sigma \varepsilon \tilde{\sigma} \sigma \alpha$ zu verstehen ist, verlangt der

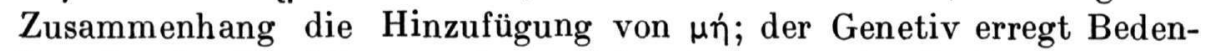
ken. Da der Begriff „vernehmen, hören" genügt, ist die Unform wohl

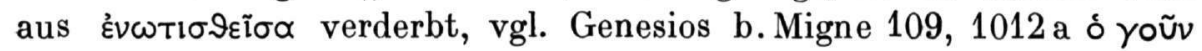

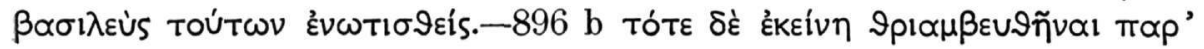

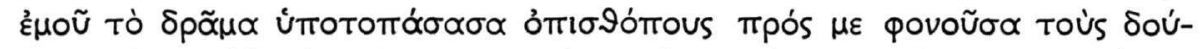

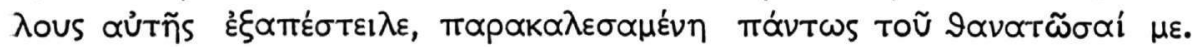
So bei Combefis zu lesen; bei Migne ist povoũ $\sigma \alpha$ in $\phi \omega \nu \circ u ̃ \sigma \alpha$ geändert. Zu schreiben ist

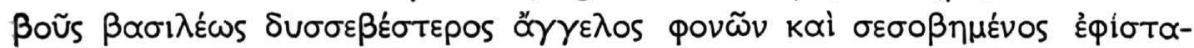

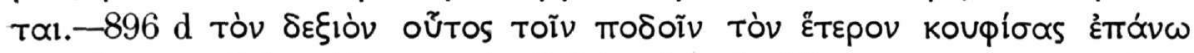
тоŨ åpı

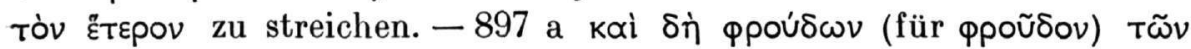

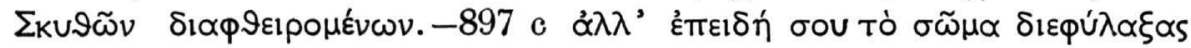

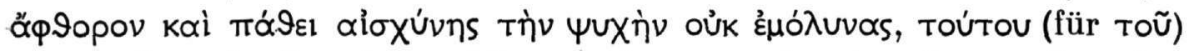

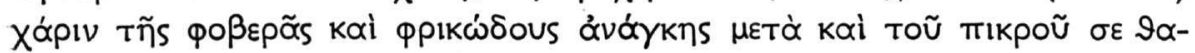

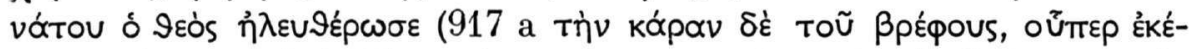
ктпขто ist тои̃ bei Migne in toútou geändert, Combefis hat richtig

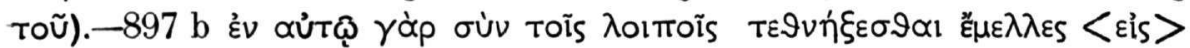

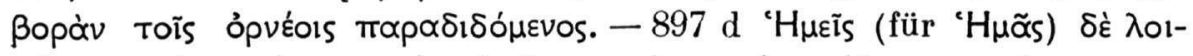

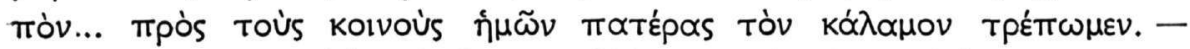

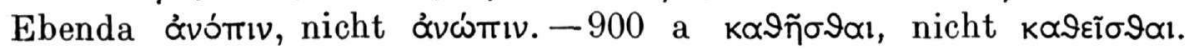

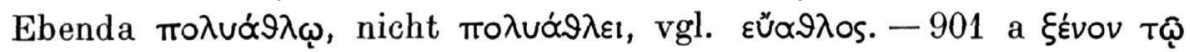

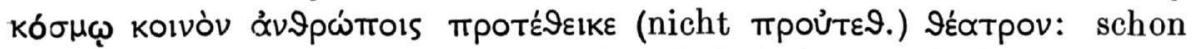

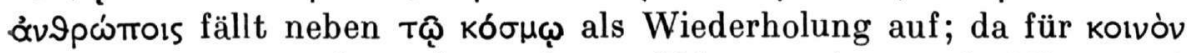

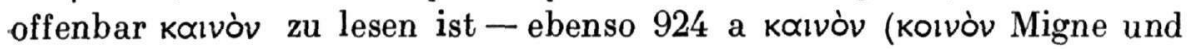

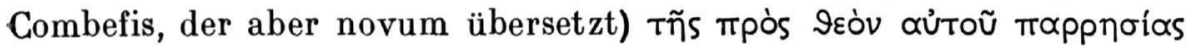

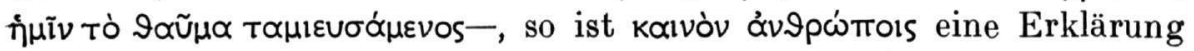

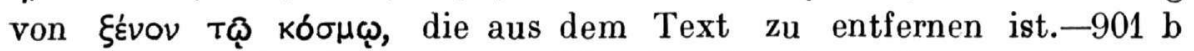

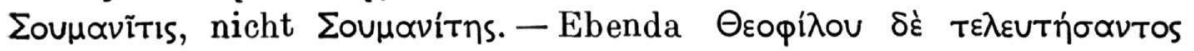

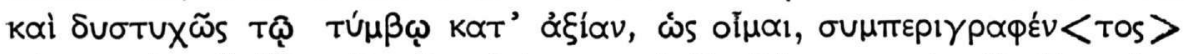

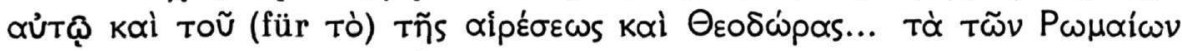

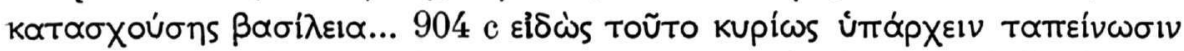

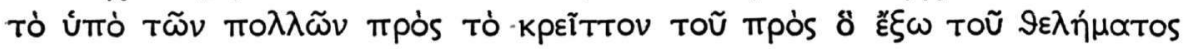

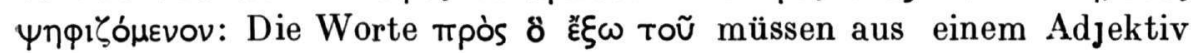
korrumpiert sein, das zu $9 \varepsilon \lambda \eta \dot{\mu} \mu$ oros Attribut ist und dessen letzte Silbe in toũ bewahrt ist; der Ueberlieferung dürfte das auch dem Sinne genügende $\pi \rho \circ \sigma \delta \circ \xi \alpha \sigma \tau o \tilde{~(, v o r g e f a ß t ") ~ a m ~ n a ̈ c h s t e n ~ k o m m e n . ~}$

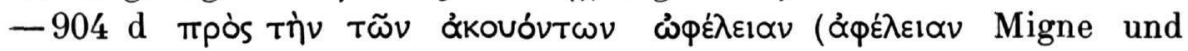

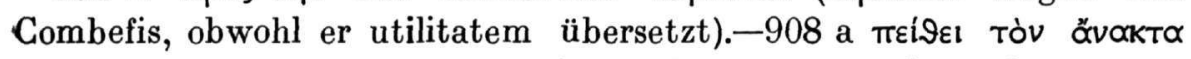

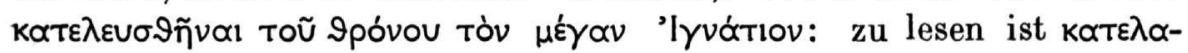

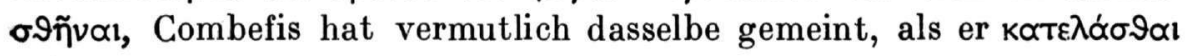




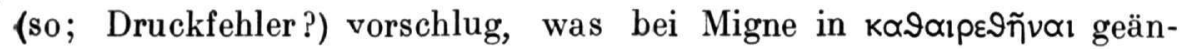

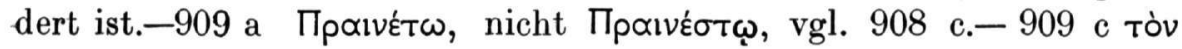

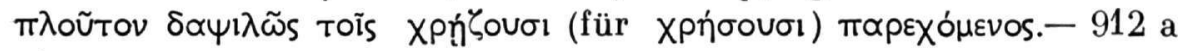

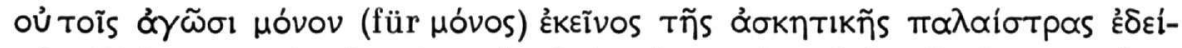

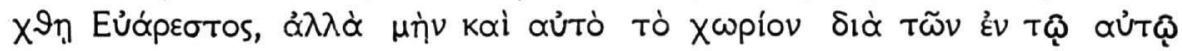

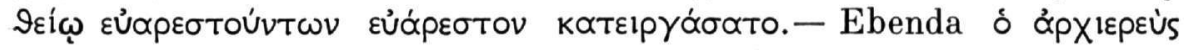

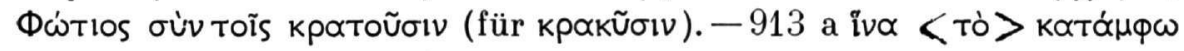

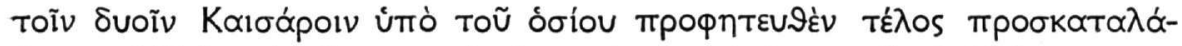

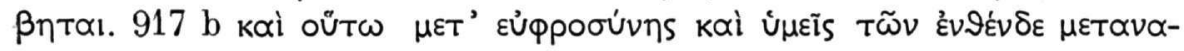

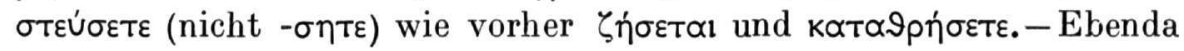

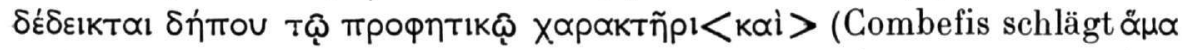

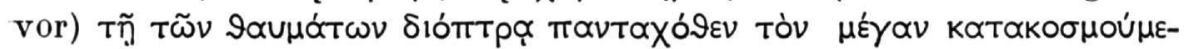

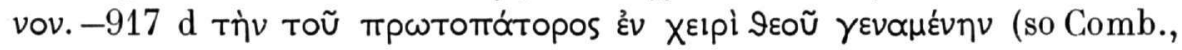

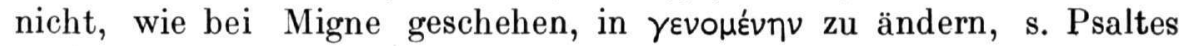

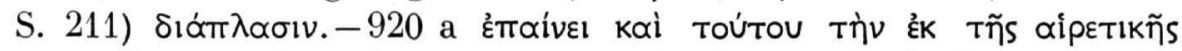

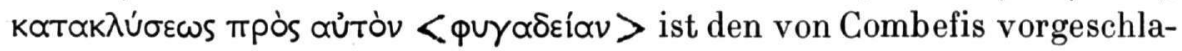

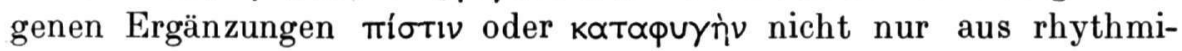

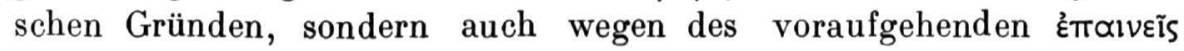

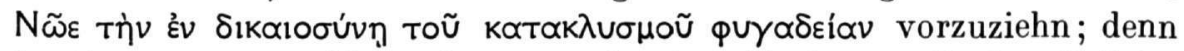
in diesem ganzen Abschnitt erstreckt der Verfasser die Parallelisierung seines Helden mit alttestamentlichen Gestalten mit Vorliebe

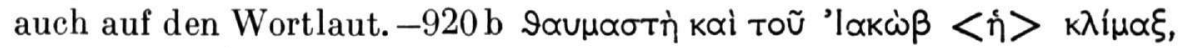

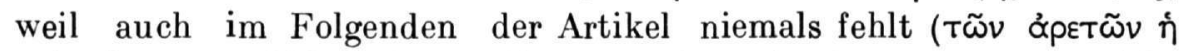

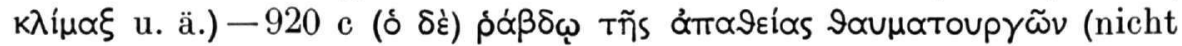

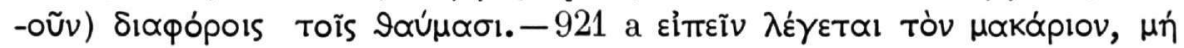

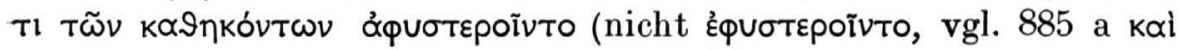

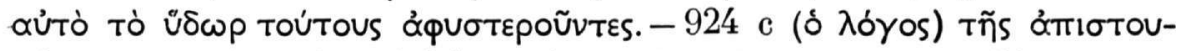

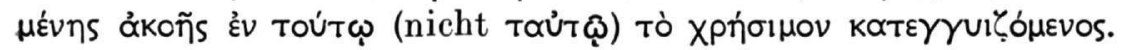

